\title{
METÁSTASIS PULMONAR SOLITARIA TRAS PROSTATECTOMÍA RADICAL
}

\author{
J. LÓPEZ FERRANDIS, J. BOÁN GARCÍA*, J. RIOJA ZUAZU, J.M. REGOJO BALBOA, \\ J.M. FERNÁNDEZ MONTERO, D. SÁNCHEZ ZALABARDO, J.M. BERIÁN POLO, \\ J.J. ZUDAIRE BERGERA, D. ROSELL COSTA, J.E. ROBLES GARCÍA
} Servicio de Urología. *Departamento de Medicina Nuclear. Clínica Universitaria.
Universidad de Navarra. Pamplona.

Actas Urol Esp. 27 (8): 637-639, 2003

\section{RESUMEN}

METÁSTASIS PULMONAR SOLITARIA TRAS PROSTATECTOMÍA RADICAL

El carcinoma de próstata es un tumor que cada vez se diagnostica en fases más precoces de su evolución pero que puede presentar un comportamiento caprichoso e impredecible. El tratamiento radical tanto prostatectomía como radioterapia es el tratamiento de elección en paciente con tumores clínicamente localizados, sin embargo el fracaso bioquímico a los 5 años de la cirugía afecta al 20-50\% de estos pacientes, la progresión a partir de los 5 años de tratamiento es un hecho poco frecuente que puede deberse a factores pronósticos poco conocidos de la propia naturaleza biológica del tumor. Presentamos un caso clínico en que tras 10 años del tratamiento radical sin evidenciar progresión bioquímica ni progresión clínica, aparece un nódulo pulmonar solitario junto con discreta elevación de las cifras de PSA.

PALABRAS CLAVE: Carcinoma de próstata. Progresión bioquímica. Progresión clínica. Metástasis pulmonar.

\section{ABSTRACT}

SOLITARY PULMONAR METASTASIS AFTER RADICAL PROSTATECTOMY

Prostate carcinoma is diagnosed in carlier phases of its evolution, bat this carcinoma may have an impredictible evolution. Radical treatment (surgery and radiotherapy) is the best treatment in clinical localitathed tumors. The biochemical failure over 5 years from the surgery is $20-50 \%$ of the patients the biochemical failure over 10 years from the surgery is a less frequent because of prognostic factors from the biologic nature of the tumor. We report a case with biochemical and clinical failure over 10 years from the surgery.

KEY WORDS: Prostate carcinoma. Biochemical failure. Clinical failure. Pulmonar metastases.

$\mathrm{E}^{\prime}$ fracaso bioquímico después de la prostatectomía radical (PR) por carcinoma de próstata (CP) localizado es del 20-25\%. En la mayoría de los pacientes ocurre durante los dos primeros años (con una media desde la cirugía hasta la progresión bioquímica de 13 meses), siendo menos común tras 5 años $(6 \%)^{1}$ y excepcional a los diez años de la cirugía. La recidiva habitualmente metastásica afecta al hueso en primer lugar. La afectación pulmonar solitaria como forma de progresión tardía de la enfermedad es un hecho aislado y peculiar. Presentamos un caso insólito en el que se realizó tratamiento mediante resección broncoscópica de metástasis pulmonar única de $\mathrm{CP}$ a los diez años de la PR. 


\section{CASO CLÍNICO}

Paciente de 73 años diagnosticado de adenocarcinoma de próstata T1c (TNM 97), Gleason 2/10 y PSA inicial de $22 \mathrm{ng} / \mathrm{mL}$. Estudios de extensión negativos. Tratado mediante PR en noviembre de 1991, con resultado anatomopatológico de adenocarcinoma de próstata Gleason 7/10, pT2b, NO, MO (TNM 97).

En sucesivas revisiones el PSA se mantiene indetectable. En noviembre de 2001 (diez años después de la cirugía) se eleva a $3,5 \mathrm{ng} / \mathrm{mL}$.

Ante la ausencia de signos de recidiva local de la enfermedad, se solicita tomografía de emisión de positrones con 18 fluoro-desoxi-glucosa (FDGPET) para estadiaje oncológico, que presenta una imagen nodular en el lóbulo inferior del pulmón derecho (Fig. 1).
Una tomografía computerizada (TC) torácica caracteriza el nódulo como compatible con neoplasia pulmonar primaria. Valorado por el departamento de cirugía torácica, se realiza resección del mismo por videotoracoscopia. El diagnóstico anatomopatológico es de metástasis pulmonar de adenocarcinoma de próstata (Fig. 2).

Acude a revisión urológica a los 25 días de la resección del nódulo pulmonar, presentando valores de PSA de 0,1 ng/mL., manteniendo en esta cifra a los 6 meses.

\section{DISCUSIÓN}

No se conoce claramente cual es la historia natural del CP. Sin embargo, hasta un 35\% de los pacientes tratados mediante PR por CP presentarán elevación de las cifras de PSA en los años

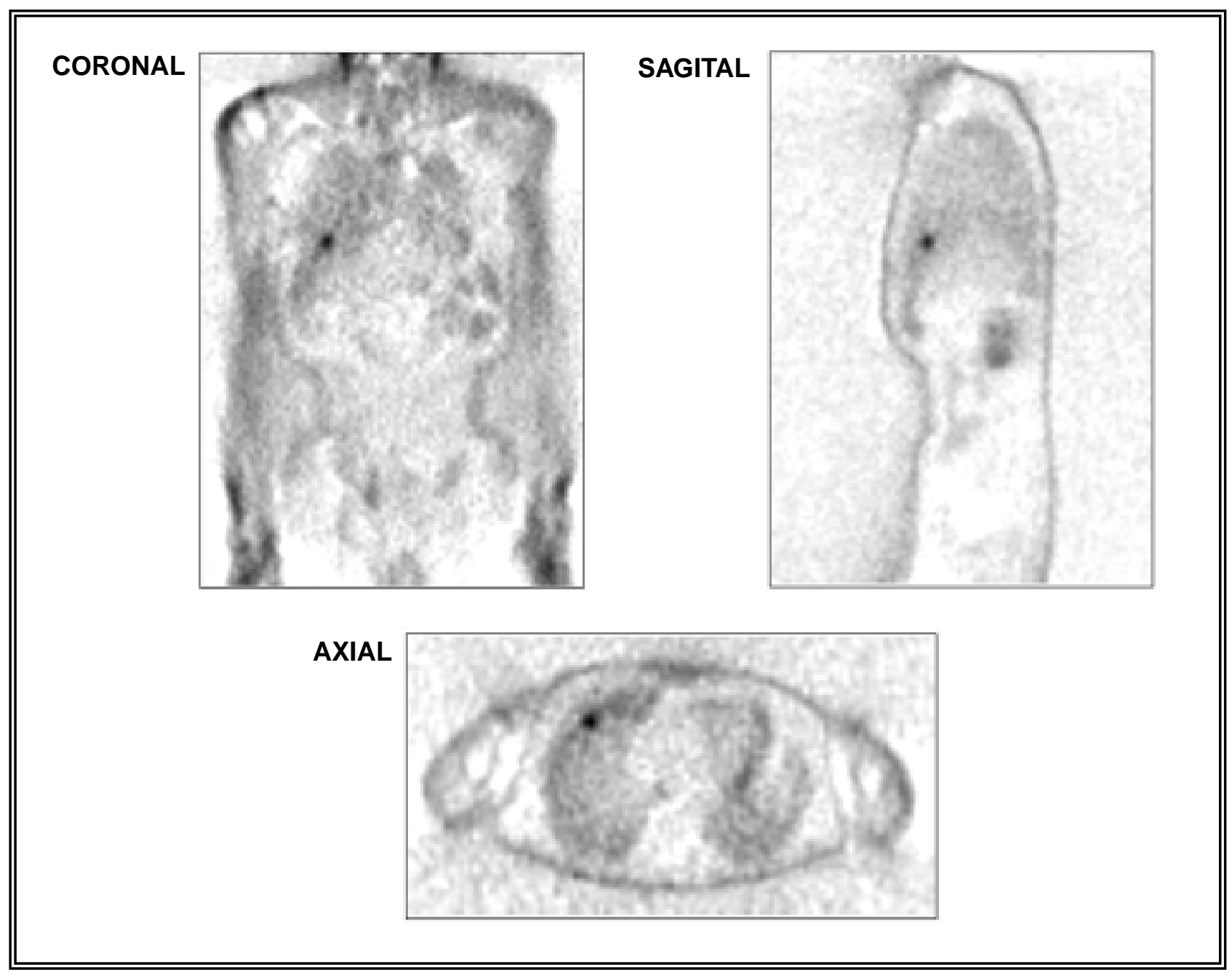

FIGURA 1. Imagen de FDG-PET donde se observa captación hipermetabólica en base de pulmón derecho. 

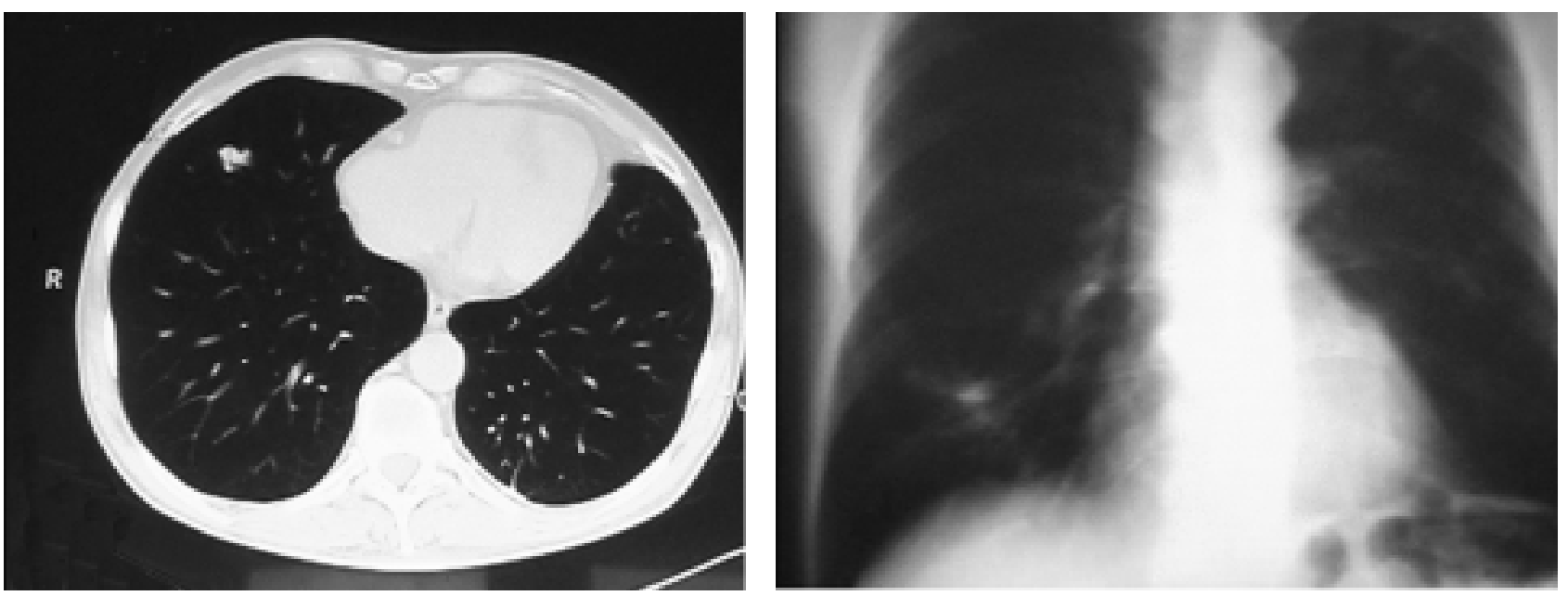

FIGURA 2. Imagen de TC de base de pulmón derecho y radiografia de tórax antero-posterior donde se observa nódulo pulmonar solitario.

siguientes a la cirugía ${ }^{2}$. La mayoría lo harán en los dos primeros años, reflejando el impacto del estadiaje clínico. Un número significativo de pacientes, incluyendo aquellos con enfermedad localizada, continuarán con progresión de la enfermedad 5 años después de la $\mathrm{PR}^{1}$. En la serie del Johns Hopkins Hospital acerca de la historia natural del CP tratado mediante PR, sólo un 4\% de los pacientes presentaron progresión bioquímica después de 10 años de la cirugía y no se menciona ningún caso de afectación pulmonar solitaria ${ }^{2}$.

Identificar la recidiva, especialmente cuando es pequeña, tardía y el PSA es menor de 10 $\mathrm{ng} / \mathrm{mL}$. es esencial, porque puede ser determinante en el posible tratamiento complementario. Sin embargo los métodos convencionales, TC, resonancia magnética y gammagrafía son frecuentemente ineficaces. En estos casos el estudio con PET puede ser de gran utilidad.

Aunque para algunos la sensibilidad no es diferente a la de la $\mathrm{TC}^{3,4}$, la capacidad para detectar metástasis en tejidos blandos es mayor. En un estudio publicado por nuestro grupo, el FDGPET detectaba la recidiva en 6 de cada 10 pacientes estudiados ${ }^{5}$.

En nuestro caso, el estudio con PET permitió un diagnóstico de localización insólito.

\section{REFERENCIAS}

1. CHRISTOPHER L. AMLING, MICHAEL L. BLUTE, ERIK J. BERGSTRALH, THOMAS M. SEAY, JEFFREY SLEZAK Y HORST ZINCKE.: Long-term hazard of progression after radical prostatectomy for clinically localized prostate cancer: continued risk of biochemical failure after 5 years. J Urol 2000; 164: 101105.

2. CHARLES R. POUND, ALAN W. PARTIN, MARIO A. EISENBERGER, DANIEL W. CHAN, JAY D. PEARSON, PATRICK C. WALSH.: Natural history of progression after PSA elevation following radical prostatectomy. JAMA 1999; 281 (17): 1591-1597.

3. SELTZER M, NARTH J, CANGIANO $\mathrm{T}$ et al.: Comparison of computer tomography (CT), positron emission tomography (PET) and monoclonal antibody scan (MAB) for evaluation of lymph node (LN) metastases in patients with PSA relapse after treatment for localised prostate cancer (CAP). Proceedings of the American Urologic Association Congress, 1998.

4. HOH CK, SELTZER MA, FRANKLIN J, DeKERNION JB, PHELPS ME, BELLDEGRUN A.: Positron emission tomography in urological oncology. J Urol 1998; 159: 347-356.

5. SANZ G, ROBLES JE, GIMÉNEZ M y cols.: Positron emission tomography with 18fluorinelabelled deoxyglucose: utility in localized and advanced prostate cancer. BJU Int 1999; 84; 1028-1031.

Dr. J. López Ferrandis

Departamento de Urología. Clínica Universitaria

C/ Pío XII, 36

31008 Pamplona (Navarra)

(Trabajo recibido el 15 octubre de 2002) 